\title{
Research on Timing Optimization of Regional Traffic Signals based on Improved Genetic Algorithm
}

\author{
Anzhi Qi \\ Liaoning Jianzhu Vocational College, Liaoyang, Liaoning, 111000
}

Keywords: Timing Optimization, Traffic Signals, Improved Genetic Algorithm

\begin{abstract}
Aiming at the problem that the signal timing schemes of the current intersections are isolated from each other and the optimization of the regional traffic control can not be effectively achieved, an optimal timing model for multi-intersection signal control schemes is proposed. The paper analyzes the characteristics of traffic flow at multiple intersections and simulates the controlled traffic flow between multiple intersections by using the cellular transmission model as a basic model. According to the characteristics of controlled parameters that affect traffic volume in multi-intersection road network and the analysis of traffic signal control accuracy and real-time requirements, an improved genetic algorithm is proposed. Combined with DIS-CO intersection numerical simulation software, the model in this paper is numerically simulated. The numerical simulation results show that the model can adapt to the change of traffic flow between intersections through time adjustment, and the average delay time of road vehicles is smaller than that of other schemes, which is an optimization model that can be adopted by real time controllers.
\end{abstract}

\section{Introduction}

As urbanization accelerates, traffic congestion has become a serious problem in many cities. There are two ways to solve the problem of traffic jam: the first is to increase the supply of urban transportation. Such methods can directly improve the urban traffic supply service through new urban roads, bridges, tunnels and other facilities. By optimizing the light control signals, improve travel rules, provide real-time traffic information and other ways to indirectly increase traffic service capabilities, thus easing travel congestion. The other is to reduce the travel demand by increasing the travel cost by reducing the travel demand of users and relying on economic control measures or administrative restrictions, so as to improve travel efficiency. As the expansion of existing urban facilities not only incurs high construction costs, but also involves social and livelihood issues such as urban planning, relocation and demolition, it is particularly important to seek cost-effective solutions to optimize existing urban traffic signal configurations. In the urban traffic control process, the regional signal control is an effective method of signal configuration, which can effectively reduce traffic jams, reduce sewage and save travel time.

\section{Regional Traffic Signal Optimization Control Model}

Targeting to minimize the average delay in regional vehicles, the modeling is a progressive process. The main optimization parameters in this model are the cycle time $\mathrm{Ci}$ of each intersection, the phase difference time PDi of each intersection and the phase time $\mathrm{Pi}$ of each intersection. The maximum period $\mathrm{Ci}$, max and the minimum period $\mathrm{Ci}$, min of each intersection are constant, but the period $\mathrm{Ci}$ of each intersection must meet the constraint:

$\mathrm{Ci}, \max >\mathrm{Ci}>\mathrm{Ci}$, mini $\in \mathrm{N}$.

Where $\mathrm{N}$ is the set of intersections in the area. In order to optimize the phase and phase time of each intersection, the cycle time of each intersection in the same area is the same, that is $\mathrm{Ci}=\mathrm{Cj}$. In traffic control, you need to provide the minimum green time to ensure traffic safety. According to HCM2000, the minimum green time GMIN is defined as: GMIN $=3.2+\mathrm{L} / \mathrm{Sp}+0.824 \times(\mathrm{Np} / \mathrm{W})$ W> 3;

GMIN = 3. $2+\mathrm{L} / \mathrm{Sp}+0.27 \times \mathrm{NpW} \leqq 3$. Where: L represents the length of the pedestrian 
crossing; Sp represents the average speed of pedestrians; W represents the width of the pedestrian crossing; $\mathrm{Np}$ is the number of pedestrians in a cycle. The phase time must be greater than or equal to the minimum green time, that is, GREENi, $\mathrm{j} \geq \mathrm{GMINi}, \mathrm{ji} \in \mathrm{N}, \mathrm{j} \in \mathrm{Pi}$.

2 adjacent junctions, the difference between the start of the coordination phase is called the phase difference. The phase difference constraint needs to take into account the up and down phases of the coordinated phase. For example, the coordinated phase is a straight-ahead phase, so the uplink of an intersection can be defined as the intersection $\mathrm{j}$ from intersection $\mathrm{i}$ to the intersection $\mathrm{j}$, while the downlink is defined as the intersection $\mathrm{j}$ To the intersection $\mathrm{i}$.

Depending on the vehicle flow saturation, the delay model in the HCM2000 uses different formulas to calculate vehicle delays in both saturated and non-saturated states. The model considers traffic flow discretization and upstream intersection control and other factors, but does not consider the phase difference; however, the phase difference plays a coordinating phase role for the adjacent intersections, so incorporating the phase difference into the delay model can further improve the traffic signal The control effect. The traffic flow is divided into two types: one is the entrance traffic flow, that is, the border traffic flow in the area, do not need to consider the phase difference; the other is the intermediate traffic flow, which is to consider the phase difference traffic flow.

\section{Genetic Algorithm Improvements}

In this paper, the constraints of traffic parameters are integrated into the coding of genetic algorithm, and the crossover and mutation processes are improved again based on the improved genetic algorithm. Finally, the optimal model is established.

In the genetic algorithm, the coding must ensure that the range of decision variables corresponds to the range of chromosome-matched genes in the genetic space. The number of decision variables can be determined according to the following rules. Let the range of decision variables be from 1 to $\mathrm{X}$. If $\mathrm{n}$ satisfies $2 \mathrm{n}-1<\mathrm{X}-1 \leq 2 \mathrm{n}$, then the range of decision variables is $\mathrm{n}$. In the decoding process, for a certain decision variable $\mathrm{W}$, if its value range is $[\mathrm{X} 1, \mathrm{X} 2]$ and the gene in the corresponding genetic space is encoded as $\{\mathrm{bkbk}-1 \mathrm{bk}-2 \ldots \mathrm{b} 1\}$ then the decoding process is $\mathrm{W}=$ $\mathrm{X} 1+\mathrm{X} 2-\mathrm{X} 12 \mathrm{k}-1 \times \Sigma \mathrm{ki}=1 \mathrm{bi} 2 \mathrm{i}-1$.

Taking one intersection as an example, the intersection is divided into main road and secondary road, as shown in below. Let 1,2,5,6 traffic flow as the main traffic flow, 3,4,7,8 traffic flow as the secondary traffic flow, 1 and 5 can be set as a phase direction, 2 and 6,3 and 7,4 and 8 are each set as a phase, ti represents the green time of traffic flow $\mathrm{i}$, tm represents the green traffic time of the main traffic flow in one cycle, tc represents the green traffic time of the secondary traffic flow in one cycle, tg is a cycle Of the phase switching time, then $\mathrm{tm}=\mathrm{t} 1+\mathrm{t} 2=\mathrm{t} 5+\mathrm{t} 6$, tc $=\mathrm{t} 3+\mathrm{t} 4=\mathrm{t} 7+\mathrm{t} 8$. Using five decision variables $\{\mathrm{f} 1, \mathrm{f} 2, \mathrm{f} 3, \mathrm{f} 4, \mathrm{f} 5\}$, the effective digits of each decision variable are 6,6,5,5,6 respectively. f1 corresponds to the period length, f2 corresponds to the main traffic flow green, f3, f4 correspond to t1, t3, f5 corresponding phase difference. According to the formula (12), the post-decoding period $\mathrm{C}=\mathrm{CMin}+(\mathrm{Cmax}-\mathrm{Cmin}) \times \mathrm{f} 1 / 63$. The minimum green time $\mathrm{tm} \min =$ MIN $\{\mathrm{t} 1 \mathrm{~min}+\mathrm{t} 2 \mathrm{~min}, \mathrm{t} 5 \mathrm{~min}+\mathrm{t} 6 \mathrm{~min}\}$ of the main traffic flow and the minimum green time tc $\min =\operatorname{MIN}\{\mathrm{t} 3 \mathrm{~min}+\mathrm{t} 4 \mathrm{~min}, \mathrm{t} 7 \mathrm{~min}+\mathrm{t} 8 \mathrm{~min}\}$ of the secondary traffic flow. According to equation (12), we can find $\mathrm{tm}=\mathrm{tm} \min +(\mathrm{tm} \max -\mathrm{tm} \min ) \times \mathrm{f} 2 / 31$, and the remaining variables can be decoded in a similar way.

The operation of genetic operators is at the heart of genetic algorithms, and the performance and effectiveness of the algorithms are largely influenced by genetic operators. In the general genetic algorithm, the crossover operator crossover probability and mutation operator mutation probability is fixed, this method is conducive to reducing the computational load of the algorithm; but with the increase of genetic algebra, it is possible to eliminate some excellent Individual, easy to fall into the local convergence, thus losing the overall effect of optimization. At present, some researches on genetic algorithms have improved the probability of crossover and mutation, and these improvements are adopted in this paper. 


\section{Example Analysis}

In this paper, we consider a typical urban network as shown above, where two parallel main lines are east-west and three parallel north-south branches. A1 and $\alpha 2$ are the starting points and $\beta 1, \beta 2$ and $\beta 3$ are the arrival points. The traveler chooses the travel route according to the principle of minimizing the travel delay, and the system configures each intersection phase time according to the principle of minimizing the total travel delay of the entire travel network. Set traffic lane capacity of 1800 units / hour, the phase switching time is 3 seconds, so a single intersection phase switching time of 12 seconds. The minimum green time for the trunk phase is 10 seconds, the maximum time is 60 seconds, the minimum green time for the feeder phase is 7 seconds, the maximum time is 40 seconds and the speed of the car is 15 meters per second. In genetic algorithms, the crossover probability is zero. 8 , the mutation probability is 0.1 , and the population size is 20 . It shows the structure of the network travel. Except for the one-way trip between the departure point and the end point, the other sections are bidirectional. Assuming that there are three lanes in the travel section, there is no interaction between different lanes. It reflects the total travel delays with genetic algebra trends, you can clearly see the advantages of genetic algorithms in the optimization process. After 120 generations have converged. It shows the average delay after smoothing with the trend of genetic algebra, reflecting the average delay of a single user in the network. It shows the distance between each travel section in this example. In determining the travel speed, the free travel time can be obtained. It shows the travel demand between the departure point and the end point. It is noted that, for generality, the default value among the OD requirements in the example is 0 for simplicity. It shows the set of travel routes under a given OD pair, and the traveler selects the travel route according to the minimum travel delay principle. During the travel process, the interaction process between the travel route and the green light time can be regarded as the game of Steinberg game. Therefore, the green light time and the path choice of the passengers finally assigned are the equilibrium solutions of this game.

It is in MATLAB 7. 0 in the calculation of the intersection of the green light distribution time and each intersection cycle. It should be noted that the actual range of the period varies from 46 seconds to 212 seconds since the range of variation of each phase is defined. The total network latency after optimization was 558260 seconds with an average delay of 156. 8 seconds. At this point, through the optimal allocation of each phase green light time in the upper layer plan, the entire network phase timing and guiding user travel can be achieved and the network delay can be minimized. As traditional algorithms face the problem of multivariate traffic network timing, there will be a sharp increase in computational complexity. As the network size increases, the computational complexity increases exponentially with the increase of variables. So difficult to achieve. Compared with the standard genetic algorithm, the improved genetic algorithm in this paper, to some extent, alleviates the computational complexity by eliminating offspring with low fitness and preserving high fitness offspring.

\section{Conclusion}

This paper uses cellular transmission model to establish DISCO-based optimization model for signalized multi-intersection trunk network. The model is used to simulate the three four-phase intersections as a collaborative optimization object, The objective function, the synergetic optimization model with the constraints of cycle, green ratio, phase difference and the number of road vehicles accommodated. Due to the requirement of real-time, the problem of slow convergence of standard genetic algorithm is improved, and the signal optimization control model of mainline multi-intersection is constructed. Numerical simulation results show that the traffic flow dynamics at the intersection of real roads can be simulated to a certain degree based on the cellular transmission model. Compared with the traditional genetic algorithm, the improved genetic algorithm has shorter searching time and the optimal value Better, with the help of this algorithm, the signal collaborative optimization control model can obtain a smaller average delay time when the input traffic changes and provide a more effective basic model for the traffic timing instrument. 


\section{References}

[1] Daganzo C F. The cell transmission model: A dynamic representation of highway traffic consistent with the hydrodynamic theory [J]. Transportation research Part B Methodological, 1994, 28(4): 269- 287.

[2] Lo H K. A Novel Traffic Signal Control Formulation [J]. Transportation research Part A, 1999, 33(6): 433-448.

[3] Lo H K, Chow A H F. Control Strategies for Oversaturated Traffic [J]. Journal of Transportation Engineering, 2004, 130(4): 466-478.

[4] Daganzo C F. The cell transmission model part II: net-work traffic [J]. Transportation Research Part B: Method-ological, 1995, 29(2) : 79- 93.

[5] Xun LI, Shiru QU, Lei CAI. Studing of PID Parameter Optimization Method for Attitude Controller of Quadrotor UAVs [J]. Journal of Computational Information Systems, 2014, 10(18) : 7805-7812. 